\title{
Medidas y responsabilidades en el sector alimentario
}

\author{
Pedro Rodríguez Guillén
}

Universidad de Sevilla

\begin{abstract}
SUMARIO. I. INTRODUGCIÓN. II. LA IMPORTANCIA DE LOS PRINCIPIOS COMUNITARIOS EN LA LEGISLACIÓN ALIMENTARIA. 1. El principio de precaución. 2. El proceso de evaluación de riesgos. 3. El sistema de APPCC como mejor forma de gestionar el riesgo. III. MARCO NORMATIVO DE LAS MEDIDAS PROVISIONALES EN EL SECTOR ALIMENTARIO. 1. A nivel comunitario. 2. A nivel nacional. IV. LA RESPONSABILIDAD PATRIMONIAL DERIVADA DE LOS DAÑOS ALIMENTARIOS GON ESPECIAL ATENCIÓN A LA COMUNIDAD DE ANDALUCÍA. 1. Reseña al régimen de responsabilidad objetiva en España. 2. El caso del aceite de orujo. 3. El caso "Magrudis". V. CONCLUSIÓN. VI. BIBLIOGRAFÍA.
\end{abstract}

RESUMEN: Nos adentramos en el relevante papel que desempeñan las autoridades públicas como garantes de que la seguridad alimentaria de los distintos productos que consumimos sea efectiva a través de su regulación mediante normas tanto internas como a nivel internacional, fundamentalmente provenientes de la UE con el famoso Reglamento 178/2002. Para saber si lo que consumimos a diario cumple con las exigencias de toda índole es preciso delimitar el régimen jurídico de las medidas asumidas por el Poder Público, preferiblemente antes de que corra riesgo la seguridad alimentaria, y no después, verificando acto seguido la intervención de las distintas administraciones en este ámbito en aras de depurar la eventual responsabilidad administrativa a la que tendrían que hacer frente nuestras autoridades si dicha intervención provoca daños o riesgos inasumibles para la población.

PALABRAS CLAVE: seguridad alimentaria, riesgos, alertas alimentarias, responsabilidad administrativa.

\section{I.INTRODUGGIÓN}

Desde la famosa alerta alimentaria conocida como el "mal de las vacas locas", la Comunidad Europea empezó a tomar acción y conciencia con la más que necesaria intervención de todas las autoridades públicas de diverso rango. Esta toma de conciencia tuvo que ver con la publicación del Libro Blanco de Seguridad Alimenta- 
ria en el año 2000, documento elaborado por la Comisión que sentó las bases de la posterior política de seguridad alimentaria que se iba a desarrollar en la UE. Aunque es verdad que este documento actúa como un mero lanzador de propuestas, no es menos cierto que supuso un punto de inflexión para el futuro de la salud pública, de los consumidores, los productores, y para la sociedad en general, con la famosa expresión "de la granja a la mesa".

El denominado "Paquete de Higiene" supuso un conjunto de Reglamentos comunitarios cuya ratio legis radicaba en no volver a lamentar una crisis alimentaria de estas características. Sin embargo, parece ser que el avance de la tecnología y la obligación de tener informado al consumidor sobre lo que come en el nuevo milenio no ha sido suficiente para evitar otras crisis alimentarias (E.coli, listeriosis, aceite de orujo). La importancia de la seguridad alimentaria en la sociedad nunca ha pasado ni pasará de moda porque la introducción de nuevos alimentos en el mercado (alimentos modificados genéticamente), los nuevos métodos de venta de alimentos en el mercado por internet y la constante codicia de los productores por abarcar la mayor cuota de mercado posible a costa de la información fraudulenta proporcionada a los consumidores está a la orden del día.

Del citado Paquete de Higiene adquiere una mayor relevancia el Reglamento (CE) $n^{\circ}$ 178/2002, del Parlamento Europeo y del Consejo, de 28 de enero de 2002, por el que se establecen los principios y los requisitos generales de la legislación alimentaria, se crea la Autoridad Europea de Seguridad Alimentaria y se fijan procedimientos relativos a la seguridad alimentaria, que será el que marcará tanto la legislación de los Estados miembros, incluido España, en materia de seguridad alimentaria por introducir importantes principios, entre los que se encuentra el de precaución (art.7), de vital importancia para nuestra investigación jurídica y del que se ha escrito mucho por autores variados, puesto que analizaremos el recurso que realizan las Autoridades Públicas a este principio en aras de evitar un daño a la población.

Esta tarea de seguridad alimentaria está encomendada a la EFSA, creada por el Reglamento 178/2002, y la AECOSAN, creada por el Real Decreto 19/2014, de 17 de enero. La Agencia Española es un organismo autónomo dependiente del Ministerio de Agricultura, Pesca y Alimentación. Los objetivos de esta agencia son: promover la seguridad alimentaria, ofreciendo garantías e información objetiva a los consumidores y agentes económicos del sector agroalimentario español; planificar, coordinar y desarrollar estrategias y actuaciones que fomenten la información, educación y promoción de la salud en el ámbito de la nutrición, y en particular, en la prevención de la obesidad ${ }^{1}$.

1 http://www.aecosan.msssi.gob.es/AECOSAN/web/agencia/seccion/sobre_aesan.htm. 
En España, al igual que en el resto de Estados miembros, la legislación nacional (Ley de Seguridad Alimentaria y Nutrición) viene inspirada en los principios y actuaciones del Reglamento 178/2002. Esta ley transcribe estos principios a nuestra legislación: principio de cautela (art.7), el de análisis de riesgos (art.5), o el de trazabilidad (art.6), a los que tendremos ocasión de analizar. Estos principios vienen a ser los mismos que los que señala el Reglamento europeo. Pero en nuestra legislación se enumeran una serie de pautas de actuación en el art.4 que seguirán las Administraciones Públicas cuando deban tomar una medida: principio de necesidad, principio de proporcionalidad, principio de no discriminación y principio de mínima afección a la competencia, los cuales se pueden reconducir al principio general de mínima intervención.

Este principio de cautela o precaución al que aludiremos fue formulado por primera vez en la Conferencia de la ONU sobre el medio ambiente y el desarrollo de 1992, más conocida como "Declaración de Río", cuyo principio 15 se formulaba en los términos siguientes: "cuando haya peligro de daño grave o irreversible, la falta de certeza científica absoluta no deberá utilizarse como razón para postergar la adopción de medidas eficaces en función de los costos para impedir la degradación del medioambiente"'?

También será relevante servirnos del principio de análisis de riesgos para deducir la responsabilidad por daños, puesto que es un sistema que se apoya en tres etapas: determinación, gestión y comunicación de los riesgos. Por lo que a nosotros nos interesa, nos centraremos en la segunda y tercera etapa, ya que es cuando deben intervenir las autoridades administrativas. No obstante, son etapas que se complementan, por lo que necesariamente la gestión y comunicación deberán seguir las directrices previamente marcadas por las autoridades sanitarias y científicas, que tendremos que conocer grosso modo a través del reflejo en la jurisprudencia comunitaria.

En la actualidad, el riesgo alimentario viene representado por el desarrollo constante y cada vez más acelerado de la denominada biotecnología alimentaria, cuya principal función viene siendo garantizar el abastecimiento general de alimentos a toda la población porque según los defensores de este conjunto de técnicas sería inviable alimentar a la población solo y exclusivamente con alimentos ecológicos, así como conferir a los alimentos un mayor grado de calidad alimentaria mediante técnicas de recombinación genética con las que se obtienen alimentos transgénicos.

En aras de una seguridad alimentaria mundial, no solo nacional, por la cada vez más internacionalización del comercio y, por ende, el gozo de una salubridad

2 https://www.un.org/spanish/esa/sustdev/agenda21/riodeclaration.htm. 
pública, que tendría que ser un hecho más que un derecho intervienen las autoridades de toda índole en el sector alimentario. Las autoridades administrativas tendrán que sustentarse en los dictámenes científicos y técnicos de las autoridades sanitarias para tomar las medidas, pero ostentando una importante discrecionalidad, como veremos.

\section{LA IMPORTANGIA DE LOS PRINGIPIOS COMUNITARIOS EN LA LEGISLACIÓN ALIMENTARIA}

\section{El principio de precaución}

El principio de precaución nos viene rubricado en el Reglamento 178/2002, art.7, en los siguientes términos:

1 .En circunstancias específicas, cuando, tras haber evaluado la información disponible, se observe la posibilidad de que haya efectos nocivos para la salud, pero siga existiendo incertidumbre científica, podrán adoptarse medidas provisionales de gestión del riesgo para asegurar el nivel elevado de protección de la salud por el que ha optado la Comunidad, en espera de disponer de información científica adicional que permita una determinación del riesgo más exhaustiva.

2. Las medidas adoptadas con arreglo al apartado 1 serán proporcionadas y no restringirán el comercio más de lo requerido para alcanzar el nivel elevado de protección de la salud por el que ha optado la Comunidad, teniendo en cuenta la viabilidad técnica y económica y otros factores considerados legítimos para el problema en cuestión. Estas medidas serán revisadas en un plazo de tiempo razonable, en función de la naturaleza del riesgo observado para la vida o la salud y del tipo de información científica necesaria para aclarar la incertidumbre y llevar a cabo una determinación del riesgo más exhaustiva.

En la legislación nacional también nos aparece regulado el principio de precaución, en concreto en la LSAN, art.7, con una dicción casi idéntica. De la interpretación que realizamos sobre estos preceptos, la consideramos parca por cuanto exige probar la efectiva existencia de efectos nocivos para la salud humana, y no meras sospechas acerca de estos efectos. Por tanto, no puede existir una incertidumbre total si quiere observar la normativa, sino solo parcial. Este "mínimo" de conocimiento, en el ámbito alimentario, deberá estar contrastada con informes objetivos y fiables que no solo provengan de una parte interesada ${ }^{3}$, como por ejemplo, los informes de la EFSA o AECOSAN.

3 MANTECA VALDELANDE, V., "El principio de precaución alimentaria", en Distribución y consumo, núm. 96 (2007), p. 94. 
Una vez que sabemos que contamos con la habilitación legal necesaria para aplicar el principio de precaución debemos conocer el modo de aplicación de este principio en el ámbito alimentario, siendo fundamental en este sentido la Comunicación de la Comisión sobre el recurso al principio de precaución ${ }^{4}$ que veremos a continuación, que será la que nos marcará las pautas para la aplicación del principio.

En un ámbito tan a la orden del día como es la ingeniería genética y la biotecnología no podría faltar su proliferación en los alimentos que consumimos a diario. "La aplicación tecnológica de las biociencias parte del supuesto [...] de la posibilidad de predecir los resultados de su intervención con una cierta probabilidad de acierto, y niegan o ignoran las incertidumbres teóricas de sus presupuestos de partida, cuantificando las posibilidades de daño y decidiendo qué riesgos deben ser evaluados" ${ }^{2}$. Aduce este autor que no es válido esgrimir una balanza costes-beneficios para la utilización de la ingeniería genética porque no se sabe siquiera cuales van a ser estos costes, por lo que la única opción que nos queda para reducir esta incertidumbre científica es la aplicación del principio de precaución para anticiparse a tal desconocimiento.

Entendiendo sobradamente que el principio de precaución solo tendrá sentido en la medida que exista un riesgo para la salud humana, ¿qué medidas podremos tomar a la sazón del principio de precaución? En la citada Comunicación, no especifica el tipo de medidas concretas, pero sí advierte que la prohibición total, por ejemplo, de la venta de un producto en el mercado puede chocar con otro principio, cual es el de proporcionalidad, pero esta medida podría ser la única factible ante un determinado riesgo. La doctrina afirma que el principio de precaución no es omnímodo ni prevalece sobre otros principios ${ }^{6}$; antes al contrario, la existencia de principios como el de proporcionalidad o legalidad, producen una relajación en su aplicación. Se puede afirmar que el principio de precaución habilita a adoptar medidas u obligaciones de medio, pero nunca de resultado por desconocerse precisamente la entidad del riesgo.

Resulta obligado analizar la STJUE de 5 de mayo de 1998, Reino Unido vs. Comisión, C-180/1996, LA LEY 54845/1998, porque fue la vez primera que se reconoció el principio de precaución como fuente del Derecho por la jurisprudencia. En este asunto, el Reino Unido impugnó la Decisión 96/239/CE, de 27 de marzo, la cual prohibía la exportación de carne bovina a países de los Estados miembros y terceros países procedente del Reino Unido como medida de protección de salud contra la encefalopatía espongiforme bovina, pero este país lo consideró una restricción a la libre circulación de mercancías. La normativa aplicada para subsumir el principio

\footnotetext{
4 https://eur-lex.europa.eu/legal-content/ES/TXT/?uri=LEGISSUM:132042.

5 SENTÍS CASTAÑO, C., "Ingeniería genética: insuficiencias teóricas y la aplicación del principio de precaución”, en Política y sociedad, vol. 39, núm. 3 (2002), p. 365.

6 IZQUIERDO CARRASCO, M. y REBOLLO PUIG, M., "El principio de precaución y la defensa de los consumidores”, en Documentación administrativa, núm. 265-266 (2003), p. 188.
} 
de precaución en el presente caso fue el art.130 TCE, el cual preveía la protección de la salud como uno de los objetivos de la política medioambiental. Fue la primera vez que se extrapoló el principio de precaución a otro sector distinto al medioambiental en aras de proteger la salud pública, de ahí su importancia.

\section{El proceso de evaluación de riesgos}

Al igual que el principio de precaución, el principio de análisis de riesgos también cuenta con asentamiento legal en las normas comunitarias en su art.6 Reglamento 178/2002. El análisis del riesgo es un proceso formado por tres elementos interrelacionados: determinación del riesgo, gestión del riesgo y comunicación del riesgo (art.3.10). La competencia sobre cada una de estas etapas o fases está encomendada a distintos sujetos y entes. Así, en la gestión deberían intervenir todos los participantes en la cadena alimentaria, no solo las autoridades administrativas: empresas alimentarias, productores o distribuidores de piensos.

En la determinación del riesgo, en cambio, solo tiene competencia la comunidad científica mediante pruebas científicas que se realizará de una manera "independiente, objetiva y transparente" (art.6.2). Por último, en la comunicación deberán colaborar tanto los que determinan el riesgo como los que lo gestionan mediante el "intercambio interactivo de información" (art.3.13), siendo esta última etapa fundamental a fin de tomar las medidas de forma coordinada y coherente entre todas las autoridades.

La determinación de riesgo, que es la parte más determinante de este proceso valga la redundancia, se lleva a cabo por la Agencia Española de Seguridad Alimentaria (AECOSAN), la cual está compuesta por un importante número de personajes científicos. Dicho organismo autónomo fue creado mediante la Ley 11/2001, de 5 de julio, por la que se crea la Agencia Española de Seguridad Alimentaria, establece que los órganos de la Agencia son el Consejo de Dirección, la Comisión Institucional, el Consejo Consultivo, el Comité Científico y el Director Ejecutivo (art.3). Su componente científico radica en la emisión de dictámenes científicos que servirán de guía de actuación a las Administraciones Públicas en el objetivo de eliminar el mayor número de riesgos posible.

Guarda especial interés la jurisprudencia comunitaria en este sentido para saber si la evaluación de riesgos se realiza correctamente en conexión con el recurso al principio de precaución. En la STJUE, Sala Cuarta, de 19 de enero de 2017, asunto Pharma vs. Alemania, C-282/2015, Ponente: Lycourgos, LA LEY 50/2017, en la cual se presenta una cuestión prejudicial sobre la compatibilidad del Derecho alemán con la legislación alimentaria europea, en concreto, con el Reglamento 178/2002 (arts.6 y 7) y el Reglamento 1925/2006. 
El Tribunal remitente se pregunta si la normativa nacional de que se trata en el litigio principal contradice el Derecho de la Unión, en la medida en que aquella normativa prohíbe con carácter general la utilización de aminoácidos en los alimentos con independencia de la cuestión de si hay suficientes razones para temer riesgos para la salud, concluyendo el mismo que la medida de excepción de autorización solo puede concederse por un período de tiempo determinado, a pesar de comprobarse la inocuidad de los alimentos. Esta medida no se rechaza por el Tribunal en base a las excesivas facultades discrecionales de las que ha hecho uso el Estado miembro porque no existe armonización legislativa comunitaria al respecto, sino por la diligencia en evaluar la información disponible.

Pero la pregunta es, ¿qué cuestiones tendrá que tomar en cuenta el poder político para aceptar un determinado riesgo existente? Siguiendo a la doctrina autorizada, es importante tanto el nivel de riesgos asumible como el tipo y grado de riesgo ${ }^{7}$. El primer obstáculo mencionado a superar viene condicionado por el escaso conocimiento de la técnica y el progreso que tienen nuestras autoridades, pero que no les quedará más remedio que "mojarse" para escoger la mejor opción. El segundo de ellos vendrá marcado por el riesgo residual, que es aquel que no está rechazado por el ordenamiento.

Por tanto, podemos concluir que la protección de la salud y las medidas que adopten las autoridades administrativas para ello dependerá de los datos científicos que tengan estas autoridades sobre este riesgo. Es decir, las autoridades científicas de un Estado miembro que cuenten con indicios sobre la existencia de un riesgo para la salud podrán adoptar estas medidas por contar con datos científicos, mientras que si en otro Estado miembro existe idéntico riesgo sobre un alimento pero no cuenta con unos mínimos datos científicos que informe sobre el riesgo, no tendrá legitimidad para tomar medida alguna.

\section{El sistema de APPGG como la mejor forma de gestionar el riesgo}

Debemos recordar que el sistema de gestión de riesgos no solo se lleva a cabo por las autoridades nacionales, sino que las empresas y productores alimentarios juegan un papel relevante en la prevención de riesgos, aunque no puedan tomar medidas provisionales mediante la denominada autorregulación. Precisamente, esta forma de gestión de riesgos por los particulares se lleva a cabo con el fin de evitar tener que tomar medidas. Me atrevería a decir que en esta prevención tienen un rol incluso más importante que los poderes públicos, ya que son al fin y al cabo los que

7 ESTEVE PARDO, J., Técnica, riesgo y derecho: tratamiento del riesgo tecnológico en el derecho ambiental, Ariel, 1999, pp. 52 y 53. 
están "en el campo de batalla" para conseguir la seguridad alimentaria. No obstante, sí hace falta que el Poder Público defina los límites de dicha autorregulación y controle "desde el exterior" la ejecución de estas medidas privadas ${ }^{8}$.

Sin embargo, la mayor parte de esta gestión del riesgo por las empresas se centra en el sistema de APPCC, al que nos referiremos aunque sea brevemente por la importancia del mismo. Es un método implantado por el Codex Alimentarius que aunque sus normas no poseen fuerza coercitiva han sido traspuestos sus principios a nuestra legislación nacional. Se basa en siete principios regulados en el art.3 de esta norma (realizar un análisis de peligros, determinar los puntos críticos de control, establecer límites críticos, establecer sistemas de vigilancia, establecer medidas correctoras cuando se observe que un punto de control crítico va a trasvasar el límite crítico establecido, establecer procedimientos de verificación para comprobar que el sistema funciona eficazmente y establecer un sistema de documentación sobre todos los procedimientos y los registros apropiados para estos principios y su aplicación).

Una parte de la doctrina, con ESTEVE PARDO a la cabeza, ve mayores problemas que ventajas en la toma de gestión del riesgo por particulares o empresas privadas porque se está trasladando cuestiones de ámbito público por ser de gran importancia a estas instancias, sin tener siquiera posibilidad la Administración de corregir las posibles decisiones que pudieran adoptar. Además, señala este autor que en países como España se encuentra menos aquilatado el sistema por haberse encontrado siempre en manos del Estado la gestión del riesgo ${ }^{9}$. Se añade que este traslado de funciones a instancias privadas o particulares no supone ni debe suponer la negación del carácter público de las mismas porque siguen estando en juego intereses constitucionales $^{10}$.

La importancia de estos principios radica en que su incumplimiento se considerará infracciones graves de carácter sanitario, cuando dicho incumplimiento depare en riesgos o daños efectivos para la salud de los consumidores, así como la inobservancia de los requerimientos de las autoridades sanitarias para el cumplimiento de estos principios (art.7.4). No nos detendremos más en esta temática porque excede del estudio de las medidas, no considerándose la implantación del mismo como una medida, sino como una forma de gestión del riesgo. Volveremos sobre este tema cuando hablemos sobre la responsabilidad administrativa para preguntarnos si una empresa podría ser responsable por no haber implantado o haber implantado de forma incorrecta el sistema.

8 ESTEVE PARDO, J., "Ciencia y Derecho ante los riesgos para la salud: evaluación, decisión y gestión”, en Documentación administrativa, núm. 265-266 (2003), p. 148.

9 ESTEVE PARDO, J. (1999: 24).

10 ESTEVE PARDO, J. (1999: 50). 
Para plasmar lo que supone realmente este sistema, describiremos de manera sucinta el control de este proceso en dos ámbitos concretos: los mataderos de cerdo ibérico para evitar enfermedades como la Salmonella y el muestreo del agua para evitar la Legionella. Por ejemplo, para controlar la Salmonella en los cerdos ibéricos, el proceso consiste en aplicar una esponja abrasiva desinfectada a cuatro partes del cerdo ibérico (pata trasera media, abdomen lateral, región dorsal media y papada), según la norma $\mathrm{ISO}^{11}$. Para el control de la Legionella en el agua, se lleva a cabo el muestreo del agua contenida en torres de refrigeración, utilizando las metodologías que marca la normativa ISO durante un período de cinco años ${ }^{12}$.

En conclusión, la mejor forma de conseguir la tan ansiada seguridad alimentaria, se puede hacer implantando un sistema de certificación de calidad alimentaria en la empresa, preferiblemente siguiendo los parámetros marcados por la norma ISO, aunque existan muchas otras como BCR o IFS que controle a su vez el correcto funcionamiento del APPCC, sin soslayar las técnicas de buenas prácticas de higiene (BPA, BPD y BPD) que se debían observar con anterioridad de la entrada de estos sistemas en la empresa.

\section{MARCO NORMATIVO DE LAS MEDIDAS PROVISIONALES EN EL SECTOR ALIMENTARIO}

\section{A nivel europeo}

Son múltiples las normas que nos podemos encontrar que habilitan a la adopción de medidas provisionales en Derecho alimentario europeo. Sin embargo, la fuente de la que emanan todas ellas se halla en el Reglamento 178/2002, en su art. 53

11 CÉSPEDES SÁNCHEZ, F. J., HERNÁNDEZ JIMÉNEZ, A., ORTEGA MARISCAL, M. A. y AMARO LÓPEZ, M.A., "El control de los criterios de higiene de los procesos en mataderos de cerdo ibérico como medio para evaluar el funcionamiento aceptable de la producción”. en M. VALERA CÓRDOBA et alt. (coords), Fornadas Ibéricas de razas autóctonas y sus productos tradicionales: innovación, seguridad y cultura alimentaria, Sevilla (2007), pp. 249-252.

12 PÉREZ VIDAL, A., TORRES LOZADA, P., HERNÁN CRUZ VÉLEZ, C., "Planes de seguridad del agua. Fundamentos y perspectivas de implementación en Colombia”, en Ingeniería e Investigación, vol. 29, núm. 3 (2009), pp. 79-85. 
sobre las medidas de emergencia ${ }^{13}$. Entre las normas que han emanado para la adopción de medidas, sobresalen aquellas que han ido dirigidas a al sector de los piensos ${ }^{14}$.

En la STJUE, Sala Tercera, de 13 Sep. 2017, asunto maíz MON 810, C-111/2016, Ponente: Bay Larsen, LA LEY 120883/2017, se interpretó el tenor de los preceptos 53 y 54. Se planteó la cuestión prejudicial acerca de si con arreglo al art.34 Reglamento 1829/2003 del Parlamento Europeo y del Consejo, de 22 de septiembre de 2003, sobre alimentos y piensos modificados genéticamente, la Comisión venía autorizada a adoptar las medidas enunciadas en el precepto objeto de análisis.

13 1. Cuando se ponga de manifiesto la probabilidad de que un alimento o un pienso, procedente de la Comunidad o importado de un país tercero, constituya un riesgo grave para la salud de las personas, de los animales o para el medio ambiente, y dicho riesgo no pueda controlarse satisfactoriamente mediante la adopción de medidas por parte de los Estados miembros afectados, la Comisión, con arreglo al procedimiento previsto en el apartado 2 del artículo 58, por iniciativa propia o a petición de un Estado miembro, adoptará de inmediato una o varias de las medidas que se exponen a continuación, en función de la gravedad de la situación:

a) si es un alimento o un pienso de origen comunitario:

i) suspensión de la comercialización o utilización del alimento en cuestión;

ii) suspensión de la comercialización o del uso del pienso en cuestión;

iii) establecimiento de condiciones especiales para ese alimento o pienso;

iv) cualquier otra medida provisional adecuada;

b) si es un alimento o un pienso importado de un país tercero:

i) suspensión de las importaciones de ese alimento o pienso procedentes de la totalidad o de parte del territorio del país tercero en cuestión y, si procede, del país tercero de tránsito;

ii) establecimiento de condiciones especiales para el alimento o el pienso procedente de la totalidad o de parte del territorio del país tercero en cuestión;

iii) cualquier otra medida provisional adecuada.

2. No obstante, en situaciones de emergencia, la Comisión podrá adoptar provisionalmente las medidas a que se refiere el apartado 1, previa consulta con el Estado o los Estados miembros afectados e informando de ello a los demás Estados miembros.

Tan pronto como sea posible, y a más tardar en un plazo de diez días hábiles, se confirmarán, modificarán, revocarán o ampliarán las medidas adoptadas, de acuerdo con el procedimiento contemplado en el apartado 2 del artículo 58, y se harán públicos sin demora los motivos de la decisión de la Comisión.

14 Así, tenemos: Reglamento de Ejecución (UE) 2018/1660 de la Comisión, de 7 de noviembre de 2018, por el que se imponen condiciones especiales a la importación de determinados alimentos de origen no animal procedentes de determinados terceros países debido a los riesgos de contaminación por residuos de plaguicidas, por el que se modifica el Reglamento (CE) n. ${ }^{\circ} 669 / 2009$ y por el que se deroga el Reglamento de Ejecución (UE) n. ${ }^{\circ}$ 885/2014; Reglamento de Ejecución (UE) 2016/6 de la Comisión, de 5 de enero de 2016, por el que se imponen condiciones especiales a la importación de piensos y alimentos originarios o procedentes de Japón a raíz del accidente en la central nuclear de Fukushima y se deroga el Reglamento de Ejecución (UE) nº 322/2014; Reglamento de Ejecución (UE) n. ${ }^{\circ}$ 884/2014 de la Comisión, de 13 de agosto de 2014, por el que se imponen condiciones especiales a la importación desde determinados terceros países de piensos y alimentos que pueden estar contaminados por aflatoxinas y se deroga el Reglamento (CE) n. ${ }^{\circ}$ $1152 / 2009$. 
Esta cuestión se ha presentado en el marco de un procedimiento penal contra el Sr. Giorgio Fidenato y los Sres. Leandro y Luciano Taboga, en el que se les imputa haber cultivado la variedad de maíz modificado genéticamente MON 810, infringiendo la normativa nacional que prohíbe este cultivo.

El Tribunal declaró que cuando no se ha demostrado que sea evidente que un producto autorizado por el Reglamento n ${ }^{\circ}$ 1829/2003 o de acuerdo con lo dispuesto en él pueda constituir un riesgo grave para la salud humana, la sanidad animal o el medioambiente, la Comisión no está obligada, en virtud del artículo 34 de dicho Reglamento, en relación con el artículo 53 del Reglamento $n^{\circ}$ 178/2002, a adoptar medidas de emergencia, en el sentido de estos artículos (apartado 28). Añadió que, aunque un Estado informe sobre la necesidad de adoptar estas medidas, si no resulta tal evidencia, la Comisión no deviene obligada, pero su falta de actuación legitima la adopción de medidas a escala nacional, así como su mantenimiento o renovación mientras que la institución comunitaria no haya adoptado alguna decisión que imponga su ampliación, modificación o derogación, según se desprende del fallo de la sentencia y de la interpretación que lleva a cabo del art.34 Reglamento 1829/2003, en relación con el art.54 Reglamento 178/2002.

Asimismo, el art.54 se rubrica bajo la expresión "Otras medidas de emergencia”. Esta norma está diseñada para el supuesto en que la Comisión no haya visto oportuno adoptar medidas de emergencia y, en tal caso, el Estado miembro podrá adoptar por iniciativa propia las denominadas medidas de protección, que solo durarán hasta que se adopten medidas comunitarias o la Comisión considere derogarlas por carecer de fundamento.

\section{A nivel estatal}

En el ámbito estatal, resalta por su importancia la LSAN, que en su Capítulo I se encarga de precisar las medidas de prevención y seguridad de los alimentos y piensos. El art.8 erige el principio de seguridad de los alimentos y piensos, el cual lo tenemos también en el art.14 Reglamento 178/2002, como principio que constituye una auténtica medida de prevención. Además, el segundo apartado se encarga de añadir que para que un alimento o pienso sea seguro, deberemos atender también a los posibles efectos o consecuencias que podría producir sobre los consumidores. En esta importante fase de prevención, juegan un papel clave los operadores económicos en la integridad de la cadena de producción mediante los denominados sistemas de control, entre los que destaca el APPCC, regulado en el art.5 Reglamento 852/2002. Cuando no hagan observar dicho sistema, el art.9 LSAN prevé dos tipos de medidas: -si un operador alimentario simplemente considera que no se cumplen algún requisito de seguridad alimentaria de los productos que pone en el mercado porque no se ha 
producido un control adecuado durante la cadena alimentaria, retirará los productos del mercado e informará a la autoridad competente sobre este hecho.

-si un operador de empresa de piensos cree que algunos de sus productos no cumplen los requisitos de inocuidad, procederá igualmente a la retirada del mercado e informará de ello a la autoridad competente. Pero además, dicho producto será destruido, a menos que la autoridad competente crea oportuno otra solución.

El art.20 LSAN, al igual que el art.53 Reglamento 178/2002, instaura las medidas de emergencia que puede tomar la Administración General del Estado cuando un riesgo alimentario no pueda controlarse de forma bastante con las medidas que pueda tomar otra autoridad competente, por ejemplo, las comunidades autónomas. Dichas medidas deberán estar basadas en una evaluación de riesgos; en su defecto, se recurrirá a la Autoridad Europea de Seguridad Alimentaria o Autoridad Española de Seguridad Alimentaria; y, por último, se aplicará el principio de cautela de no existir ningún dato científico.

Extraemos de esta norma que la autoridad estatal siempre intervendrá en última instancia cuando no sea posible conseguir la seguridad a instancias de otra autoridad y, si interviene, siempre habrá de basarse en datos científicos. Como señala TOLEDO MARTÍN, estas medidas que se adopten tendrán que cumplir los requisitos establecidos por el art.4 LSAN (principio de necesidad, proporcionalidad, no discriminación y mínima afección a la competencia $)^{15}$.

También contamos en nuestro territorio patrio con la Ley 12/2013, de 2 de agosto, de medidas para mejorar el funcionamiento de la cadena alimentaria, que tiene como finalidad "mejorar el funcionamiento y la vertebración de la cadena alimentaria de manera que aumente la eficacia y competitividad del sector agroalimentario español y se reduzca el desequilibrio en las relaciones comerciales entre los diferentes operadores de la cadena de valor, en el marco de una competencia justa que redunde en beneficio no sólo del sector, sino también de los consumidores" (E.M.III). Vemos, por tanto, que con una clara inspiración comunitaria, la adopción de medidas antes que se constate un riesgo es mucho más preferible que hacerlo cuando ya se ha producido.

La LGSP se dictó con el esfuerzo, no solo de garantizar la sanidad, que ya hacía de antaño la Ley General de Sanidad, sino también con el ánimo de tomar una actitud activa la Administración Pública en aras de mantener "el mayor nivel de salud posible a través de las políticas, programas, servicios, y en general actuaciones de toda índole desarrolladas por los poderes públicos, empresas y organizaciones ciu-

15 TOLEDO MARTÍN, A., Las medidas provisionales en el Derecho alimentario, tesis doctoral, Universidad de Navarra (2012), p. 329. 
dadanas con la finalidad de actuar sobre los procesos y factores que más influyen en la salud, y así prevenir la enfermedad y proteger y promover la salud de las personas, tanto en la esfera individual como en la colectiva" (art.1). Entre los factores que han de tener en cuenta las autoridades gubernativas para vigilar el nivel de salud pública en la población se encuentra, como no podía ser de otro modo, la seguridad alimentaria y los riesgos alimentarios $\left(\operatorname{art} \cdot 12 \cdot 2 \cdot 3^{\circ}\right)$.

El art.54 LGSP sanciona las medidas especiales y cautelares que las autoridades están legitimadas a adoptar que, en definitiva, son también medidas de urgencia o extraordinarias cuando el riesgo para la salud pública sea inminente. A diferencia de la LSAN, el apartado segundo de este precepto establece un elenco de medidas que en todo caso son numerus apertus. Las medidas que se adopten deberán respetar los principios generales de acción en salud pública del art.3 (equidad, salud en todas las políticas, pertinencia, precaución, evaluación, transparencia, integralidad y seguridad).

Entre estas se encuentran: la inmovilización y, si procede, el decomiso de productos y sustancias; la intervención de medios materiales o personales; el cierre preventivo de las instalaciones, establecimientos, servicios e industrias; la suspensión del ejercicio de actividades; la determinación de condiciones previas en cualquier fase de la fabricación o comercialización de productos y sustancias, así como del funcionamiento de las instalaciones, establecimientos, servicios e industrias a que se refiere esta ley, con la finalidad de corregir las deficiencias detectadas; cualquier otra medida cuando existan indicios racionales de riesgo para la salud.

Las medidas "preventivas" a las que alude la LGS aparecen en los siguientes términos, en el art.26:

1. En caso de que exista o se sospeche razonablemente la existencia de un riesgo inminente y extraordinario para la salud, las autoridades sanitarias adoptarán las medidas preventivas que estimen pertinentes, tales como la incautación o inmovilización de productos, suspensión del ejercicio de actividades, cierres de Empresas o sus instalaciones, intervención de medios materiales y personales y cuantas otras se consideren sanitariamente justificadas.

2. La duración de las medidas a que se refiere el apartado anterior, que se fijarán para cada caso, sin perjuicio de las prórrogas sucesivas acordadas por resoluciones motivadas, no excederá de lo que exija la situación de riesgo inminente y extraordinario que las justificó.

CALLEJA REINA, basándose a su vez en la doctrina constitucional, fija los requisitos que debería cumplir una medida para considerar que está constitucio- 
nalmente justificada ${ }^{16}$ : que se persiga un fin constitucionalmente legítimo; que su adopción se encuentre amparada por una norma de rango legal (principio de legalidad); que sea acordada judicialmente, pero sin descartar que la ley pueda habilitar a otros sujetos por razones de urgencia o necesidad; motivación de la resolución que la acuerde; y, finalmente, proporcionalidad de la medida de manera que el sacrificio que la medida, idónea y necesaria a los fines constitucionalmente legítimos que se pretenden, no implique un sacrificio desmedido. Obviamente, la condición de autorización judicial exigida por el Tribunal Constitucional carecerá de sentido porque en la mayoría de los casos este tipo de medidas se adoptan en situaciones de urgencia, sin perjuicio de que sean vean sometidas a control judicial a posteriori a través de la ratificación por los Juzgados de lo Contencioso-Administrativo (art.8.6 LJCA).

\section{LA RESPONSABILIDAD PATRIMONIAL DERIVADA DE LOS DAÑOS ALIMENTARIOS GON ESPEGIAL ATENGIÓN A LA CO- MUNIDAD AUTÓNOMA DE ANDALUGÍA}

\section{Reseña al régimen de responsabilidad objetiva en España}

La responsabilidad de la Administración Pública se ubica en el Capítulo IV de la LRJSP. El principio de responsabilidad patrimonial viene preceptuado de forma objetiva, en los siguientes términos:

Los particulares tendrán derecho a ser indemnizados por las Administraciones Públicas correspondientes, de toda lesión que sufran en cualquiera de sus bienes y derechos, siempre que la lesión sea consecuencia del funcionamiento normal o anormal de los servicios públicos salvo en los casos de fuerza mayor o de daños que el particular tenga el deber jurídico de soportar de acuerdo con la Ley (art.32.1).

La objetividad del régimen proviene nada más y nada menos directamente del mandato constitucional contenido en el art.106.2 CE:

Los particulares, en los términos establecidos por la ley, tendrán derecho a ser indemnizados por toda lesión que sufran en cualquiera de sus bienes y derechos, salvo en los casos de fuerza mayor, siempre que la lesión sea consecuencia del funcionamiento de los servicios públicos.

En el sector alimentario, que es del que nos estamos ocupando, guarda especial interés la responsabilidad concurrente de las Administraciones Públicas, es decir,

16 CALleja REINA, M. A., "Contagio del ébola en España: ejemplo de cómo no comunicar una crisis”, en C. MATEOS MARTÍN y F.J. HERRERO GUTIÉRREZ (coords), La pantalla insomne, 2016, pp. 83-99. 
cuando nos encontramos con más de una Administración Pública responsable de los daños ocasionados, art.33:

1. Cuando de la gestión dimanante de fórmulas conjuntas de actuación entre varias Administraciones públicas se derive responsabilidad en los términos previstos en la presente Ley, las Administraciones intervinientes responderán frente al particular, en todo caso, de forma solidaria. El instrumento jurídico regulador de la actuación conjunta podrá determinar la distribución de la responsabilidad entre las diferentes Administraciones públicas.

2. En otros supuestos de concurrencia de varias Administraciones en la producción del daño, la responsabilidad se fijará para cada Administración atendiendo a los criterios de competencia, interés público tutelado e intensidad de la intervención. La responsabilidad será solidaria cuando no sea posible dicha determinación.

Como afirma la doctrina, en un Estado descentralizado como el nuestro en el que las competencias están repartidas por la Constitución española entre el Estado central y las comunidades autónomas, y aún más en sede de salud pública y protección de los consumidores y usuarios, se tornaba en esencial un precepto como éste que arbitrara la posible responsabilidad. No obstante, la responsabilidad solo será solidaria entre las distintas Administraciones Públicas cuando no se cuente con un instrumento jurídico que dirima claramente la cuestión ${ }^{17}$.

Para concretar aún más la administración responsable en caso de producirse una alerta alimentaria habrá que oír sobre todo a la EFSA y AECOSAN porque estas instituciones corporativas son las que tienen un registro sanitario de empresas y alimentos, por lo que en caso de producirse una alerta con indicios de una posible Administración responsable detrás de la alerta, solo habrá que comprobar en la Comunidad Autónoma en la que se sitúa esta empresa o alimento. No obstante, como veremos, es muy fácil que haya una empresa responsable, pero es más difícil que se pueda objetivar la responsabilidad a una Administración Pública.

En cuanto a la indemnización, que va a ser la forma de resarcir los daños y perjuicios a los perjudicados, resulta de vital importancia el criterio de "estado de la ciencia o de la técnica" para un estudio como el nuestro porque nos marcará la frontera de lo indemnizable y lo no indemnizable. Por lo cual, no serán indemnizables "los daños que se deriven de hechos o circunstancias que no se hubiesen podido prever o evitar según el estado de los conocimientos de la ciencia o de la técnica existente en el momento de producción de aquellos, todo ello sin perjuicio de las prestaciones asistenciales o económicas que las leyes puedan establecer para estos casos" (art.34.1).

17 CAMPOS ACUÑA, M, C., Comentarios a la Ley 40/2015 de Régimen Furídico del Sector Público, El Consultor de los Ayuntamientos, 2017. 
Sobre el concepto de estado de la ciencia y de la técnica volveremos más adelante porque fue introducido como novedad para esta Ley.

Sea como fuere, los requisitos para que el daño sea indemnizable son: daño antijurídico, efectivo, evaluable económicamente e individualizable. El requisito de la antijuricidad, en el que podemos englobar el deber jurídico de soportar el daño y el estado de los conocimientos científicos y técnicos, aparece ahora mencionado en el art.32, mientras que en el régimen anterior aparecía mencionado junto al requisito de la indemnización ${ }^{18}$.

Para analizar la cláusula "estado de los conocimientos científicos y técnicos", tomaremos como referencia la Directiva 85/374/CEE, del Consejo, de 25 de julio de 1985, relativa a la aproximación de las disposiciones legales, reglamentarias y administrativas de los Estados miembros en materia de responsabilidad por los daños causados por productos defectuosos, cuyo art.1 establece que el productor será responsable de los daños causados por los defectos de sus productos. El art.6 nos aclara que un producto es defectuoso "cuando no ofrece la seguridad a la que una persona tiene legítimamente derecho". Esta norma se ha integrado en nuestro Derecho positivo en el Libro tercero, Título II, Capítulo I del Real Decreto Legislativo 1/2007, de 16 de noviembre, por el que se aprueba el texto refundido de la Ley General para la Defensa de los Consumidores y Usuarios y otras leyes complementarias.

Sin embargo, el quid iuris se centra en la exoneración de responsabilidades al productor en los casos tasados en el art.140 LGDGU, cuya prueba corresponde al productor. Siguiendo a PRIETO-MOLINERO, estas exoneraciones no suponen una excepción al régimen de responsabilidad objetiva, puesto que sería injusto hacer responsable al productor, entre otras razones, cuando no se busca un beneficio económico, el producto se ajusta a las normas imperativas dictadas por los poderes públicos o que el defecto venga motivado del propio diseño del producto. La excepción al régimen lo constituyen los denominados "riesgos de desarrollo", lo cual fue derogado, que supone una verdadera inversión al régimen de responsabilidad objetiva, es decir, se hace responder a estos fabricantes y productores a pesar de no conocer dichos riesgos según el estado de la ciencia y de la técnica.

Entre los argumentos que maneja el autor para oponerse a esta eliminación de la causa, encontramos ${ }^{19}$ : por un lado, los riesgos de desarrollo no permiten conocer mediante datos científicos la relación de causalidad entre el daño y el producto; por otro lado, el perjuicio para los consumidores que solo podrán obtener productos bási-

18 GARCÍA RUBIO, F. y FUENTES I GASÓ, J., La responsabilidad de las administraciones públicas tras la nueva ley de régimen jurídico del sector público. Cuatro estudios, Atelier, 2017, p. 58.

19 PRIETO MOLINERO, R. J., "La excepción por estado de la ciencia en España: ¿un camino hacia la innovación segura?", en Práctica de Derecho de Daños, núm. 51 (2007). 
cos que no son sometidos a técnicas de progreso y mejora: y, por último, se convierte el sistema en una especie de asegurador universal a lo que no está llamado, según ha repetido en varias ocasiones la jurisprudencia.

Otro sector doctrinal, por el contrario, ha criticado duramente la cláusula "estado de los conocimientos científicos y técnicos" en base a: por un lado, el mandato constitucional del art.106.2 GE solo prevé como causa de exoneración de responsabilidad la fuerza mayor, por lo que se estarían añadiendo causas de exención por una vía distinta a nuestra Carta Magna; por otro lado, que los centros públicos estarían más privilegiados que los centros privados, por cuanto en éstos últimos no entraría en juego dicha causa de exoneración, conculcando ello el principio de igualdad del art.14 $\mathrm{CE}^{20}$.

Hay que dejar claro que esta cláusula a la que hacemos referencia la estamos estudiando porque está prevista para el ámbito de la responsabilidad patrimonial de la Administración, tema que nos ocupa. Sin embargo, ésta es también utilizada para la responsabilidad civil de los productos defectuosos, en cuanto la apreciación de responsabilidad de los productores por daños a los consumidores suele ser más frecuente que la responsabilidad patrimonial de la Administración debido a que, sin ánimo de entrar en un profundo análisis, las exigencias de los Tribunales civiles para constatar la responsabilidad son más suaves que las tenidas en cuenta por los Tribunales contenciosos.

\section{El caso del aceite de orujo}

Entrando más de lleno en la casuística de las alertas alimentarias producidas en nuestro país, nos encargamos ahora de analizar desde el punto de vista jurídico aquellos aspectos más relevantes que supuso esta crisis sanitaria.

Esta alerta alimentaria fue declarada por la Consejería de Salud de Andalucía, a través de Resolución de 3 de diciembre de 2001, a raíz del descubrimiento de HAPs en una serie de partidas de aceite de orujo refinado de oliva y aceite de orujo de oliva. Dicha alerta alimentaria consistió básicamente en una medida: la inmovilización cautelar de dichas partidas de aceite a fin de evitar su puesta en el mercado. Esta inmovilización cautelar estaría vigente hasta descartar la presencia de este compuesto mediante un método analítico adecuado y con un límite numérico determinado. La constatación de estas sustancias en el aceite de orujo traía como causa la introducción de nuevas tecnologías en los procesos de elaboración. En cualquier caso, esta sustancia excedía el límite establecido tanto en la normativa (Orden ministerial de 25

20 BUStO LAGO, J. M., "La responsabilidad civil de las Administraciones Públicas", en L. F. REGLERO CAMPOS y J. M. BUSTOS LAGO (coords.), Tratado de Responsabilidad Civil. Aranzadi, 2014, pp. 58 y 59 (recuperado de Aranzadi Digital). 
de julio de 2001) como en el informe emitido por la Agencia Española de Seguridad Alimentaria.

A lo largo de este apartado, veremos las distintas resoluciones judiciales y normativas aplicadas que han gravitado alrededor del caso. Nos detendremos, en primer orden, en la STS, Sala Tercera, de lo Contencioso-administrativo, Sección $4^{\mathrm{a}}$, de 14 Nov. 2007, Rec. 77/2004, Ponente: Picó Lorenzo, LA LEY 193699/2007, en la que se discute sobre si la inmovilización cautelar acordada por la Administración andaluza en el caso del aceite de orujo debió seguir la instrucción de un expediente por la vía de urgencia y con trámite de audiencia a la parte interesada o, dada la particularidad de esta alerta alimentaria, se hacía prescindible dicho procedimiento.

La Sala argumenta que las medidas provisionales del antiguo art.72 Ley 30/1992 se pueden adoptar en los casos de urgencia y antes de la iniciación del procedimiento administrativo siempre que lo habilite una norma con rango de ley $\left(\mathrm{FJ} 5^{\circ}\right)$. Esta norma con rango de ley viene constituida por el art.26 LGS que, según se interpreta por el Tribunal y se fija como doctrina legal, "el art. 26 de la LGS habilita a las autoridades sanitarias competentes para adoptar las medidas en él contempladas sin necesidad de la instrucción previa de un procedimiento administrativo, cuando resulten necesarias para garantizar la protección de la salud de los consumidores". No obstante, esta doctrina legal no es fijada tampoco de forma ilimitada porque aduce la Sala que la responsabilidad patrimonial derivada de estas medidas para el particular tendrá que ser valorada ad casum y sin que el principio de precaución constituya una causa eximente de responsabilidad porque el art.26 solo encarga de habilitar una actuación administrativa en una situación de extrema gravedad, pero la cual podría dar lugar perfectamente a responsabilidad administrativa $\left(\mathrm{FJ} 6^{\circ}\right)$.

Por otra parte, en la STS, Sala Tercera, de lo Contencioso-administrativo, Sección 4 $4^{\text {a }}$, de 27 Jun. 2007, Rec. 10820/2004, Ponente: Martínez-Vares García, LA LEY 79372/2007, el Tribunal valora en el recurso de casación la prueba de instancia acerca de si concurren los requisitos necesarios para adoptar la medida de inmovilización cautelar. Estos dos requisitos son la existencia o la sospecha razonable de un riesgo inminente y extraordinario para la salud, según establece la Directiva 92/59/CEE del Consejo de 29 de junio de 1992, relativa a la seguridad general de los productos. La Sala valora que las pruebas arrojaron, efectivamente, la existencia de concentraciones de HAP en el aceite de orujo susceptibles de entrañar riesgos para la salud humana, pero que tales riesgos no eran inminentes ni extraordinarios a juicio del Ponente. Además, afirma que la sentencia recurrida no tuvo suficiente motivación porque juzgó con datos que no exigían una medida tan drástica como la inmovilización cautelar. Se esgrimió también que estas sustancias se mantuvieron por 
debajo de los niveles considerados nocivos para la salud, según la Orden ministerial del Gobierno. Esta sentencia, por tanto, anuló la alerta alimentaria decretada $\left(\mathrm{FJ} 5^{\circ}\right)$.

Entrando más de lleno en lo que hace a responsabilidad patrimonial, pasaremos a ofrecer el análisis de la Sentencia de 4 Mar. 2009, cuya línea argumentativa ha sido seguida por las Sentencias de 28 May. y 8 Oct. 2010 y 16 Sept. 2011 del Tribunal Supremo. En la STS, Sala Tercera, de lo Contencioso-administrativo, Sección 6 ${ }^{a}$, Sentencia de 4 Mar. 2009, Rec. 9520/2004, Ponente: Puente Prieto, LA LEY 14517/2009, se litigia si a consecuencia de la revocación de la alerta alimentaria que efectuó la STS, Sala Tercera, de lo Contencioso-administrativo, Sección $4^{\mathrm{a}}$, de 27 Jun. 2007, Rec. 10820/2004, Ponente: Martínez-Vares García, LA LEY 79372/2007, por la anulación de la Resolución de 3 de julio de 2001, se produjo unos daños a la empresa Extractora del Genil S.A. que no tenía el deber jurídico de soportar.

La Sala argumenta para negar la antijuricidad del daño y, por ende, negar la indemnización a la recurrente que, antes de declararse la alerta el 3 de julio de 2001, "existían ya informes anteriores a nivel internacional que ponían de manifiesto los posibles riesgos existentes para la salud, así como que la citada alerta se produjo, no como consecuencia de una simple actuación de oficio de las autoridades sanitarias españolas, sino provocada por la difusión en medios de comunicación de la República Checa sobre el peligro del consumo de aceite procedente de España al ser susceptible a largo plazo de producir células carcinógenas, a la vista de lo cual se procedió a la práctica de análisis aleatorios". Se ratifica que "la inmediatez entre la alerta y la Orden ministerial que determinó los niveles máximos de la sustancia, 22 días, durante los que se realizaron los análisis descritos y se fijaron las bases para la orden de referencia, demuestra la diligencia de la Administración y la no antijuricidad del daño" (FJ $\left.4^{\circ}\right)$.

Finalmente, se señala que la medida acordada por la Administración tuvo su FJ en el RD 44/1996, de 19 de enero, que regula en su art.3 las obligaciones de productores y distribuidores, imponiéndoles el cumplimiento de la obligación de comercializar únicamente productos seguros, y obligando a los productores a tomar medidas apropiadas para mantener informados a los consumidores de los riesgos que los productos que comercialicen podrían presentar. Así, la medida se adoptó a consecuencia de una actuación de la empresa productora del aceite que no puso todos los medios necesarios para que el producto fuera seguro, y no derivado de un peligro natural que contenía el producto, por lo que dicha medida supone un daño que tendrá que soportar legítimamente $\left(\mathrm{FJ} 4^{\circ}\right)$.

Hemos visto y concluimos para el caso del aceite de orujo que la anulación de una medida por la vía judicial no siempre da lugar a responsabilidad administrativa. 
Aprecio, a título personal, que la cláusula ya argüida "estado de los conocimientos científicos y técnicos" no es siquiera mencionada de pasada en ninguna de las resoluciones judiciales que se han ocupado de resolver el caso del aceite de orujo, pero que si tenemos en cuenta que la crisis fue producida por la introducción de una nueva tecnología en el proceso de elaboración del aceite, sería también subsumible para eximir de responsabilidad administrativa. Como expone acertadamente TOLEDO MARTÍN, cuanto mayor sea la urgencia por los daños inminentes que los riesgos pudieran producir, menos justificación científica y técnica tendrá que realizar la Administración para adoptar la medida ${ }^{21}$.

\section{El caso "Magrudis"}

En este apartado describiremos someramente en que consistió la enfermedad que desencadenó la alerta alimentaria, trazaremos la legislación a tener en cuenta y aludiremos alguna resolución judicial de trascendencia en este punto. Las listeriosis es una enfermedad zoonótica transmisible a los humanos provocada por el consumo de alimentos que poseen la bacteria L.monocytogenes. Constituye una obligación de los operadores alimentarios controlar en todas las cadenas de producción la ausencia de este patógeno.

Las normas con las que contamos se han hecho ad hoc para la enfermedad, como el Reglamento (UE) 2015/538, de 31 Marzo (modifica anexo II del Reglamento $1333 / 2008$ respecto al uso de ácido benzoico y benzoatos (E 210-213) en los camarones cocidos conservados en salmuera), en la que se establece que como consecuencia de la notificación de 198 muertes en la Unión, la Comisión determinó un límite máximo de $1000 \mathrm{mg} / \mathrm{kg}$ de ácido benzoico y benzoatos (aditivos) en los camarones cocidos conservados en salmuera.

Más longeva es la Directiva 2003/99/CE del Parlamento Europeo y del Consejo, de 17 de noviembre de 2003, sobre la vigilancia de las zoonosis y los agentes zoonóticos y por la que se modifica la Decisión 90/424/CEE del Consejo y se deroga la Directiva 92/117/CEE del Consejo, cuyo objeto se concreta en asegurar la adecuada vigilancia de las zoonosis, los agentes zoonóticos, y la resistencia a los antimicrobianos asociada, así como la debida investigación epidemiológica de los brotes de enfermedades transmitidas por los alimentos, de forma que pueda recogerse en la Comunidad la información necesaria para evaluar las tendencias y las fuentes pertinentes, y su ámbito de aplicación en la vigilancia, investigación e intercambio de información (art.1). Entre las zoonosis objeto de vigilancia se encuentra, como no podía ser de otro modo, la listeriosis.

\footnotetext{
21 TOLEDO MARTÍN, A. (2012: 488).
} 
Como es obvio, la jurisprudencia comunitaria siempre apuesta por la protección de la salud si así lo ven oportuno los Estados miembros. En la STJUE, Sala Quinta, de 24 Oct. 2002, asunto C-121/00, Ponente Jann, LA LEY 221191/2002, se plantea una cuestión prejudicial por el tribunal alemán sobre la interpretación de la Directiva 91/493/CEE del Consejo, de 22 de julio de 1991, por la que se fijan las normas sanitarias aplicables a la producción y a la puesta en el mercado de los productos pesqueros, en relación si los arts. 28 y 30 TCE admiten un nivel de tolerancia cero en cuanto a la presencia de la Listeria monocytogenes en productos pesqueros no conservados por medios químicos. Se discute si la presencia de listeriosis en $25 \mathrm{gr}$. del pescado habilita a restringir en el comercio intracomunitario dicho producto o sería una medida desproporcionada.

Siguiendo jurisprudencia anterior (sentencias de 16 de abril de 1991 Comisión/Alemania, apartado 41, y EurimPharm, C-347/89, apartado 26) según los datos científicos disponibles sobre la listeriosis, que no permiten fijar con certeza la concentración precisa de agentes patógenos de Listeria monocytogenes más allá de la cual un producto a base de pescado representa un peligro para la salud humana, corresponde a los Estados miembros, a falta de armonización en la materia, decidir el nivel que pretenden garantizar de protección de la salud y vida de las personas, siempre que se tengan en cuenta las exigencias de la libre circulación de mercancías (apartado 38).

En el ámbito estatal, hemos de señalar que juega un papel clave la Red Nacional de Vigilancia Epidemiológica, creada mediante RD 2210/1995, de 28 de diciembre. Este organismo dependiente del Ministerio de Sanidad permite la recogida y el análisis de la información epidemiológica con el fin de poder detectar problemas, valorar los cambios en el tiempo y en el espacio, contribuir a la aplicación de medidas de control individual y colectivo de los problemas que supongan un riesgo para la salud de incidencia e interés nacional o internacional y difundir la información a sus niveles operativos competentes (art.1 RD).

En Andalucía, en agosto y septiembre de 2019, saltaron varias alertas por listeria, algunas de las cuales tuvieron consecuencias muy graves. Para hacer frente a esta crisis, el gobierno andaluz aprobó en septiembre de ese año un Plan de Choque Extraordinario contra esta enfermedad con fecha de 27 de septiembre de 2019. Este vademécum de medidas trata de que sean los propios operadores alimentarios, a través del famoso APPCC, los que asuman los riesgos de la puesta en el mercado de los distintos alimentos al consumidor y definan su política alimentaria, dentro de los límites de control que ostentan las autoridades.

Para asegurar la salud de todos los consumidores y la inocuidad de los alimentos se establecen las siguientes pautas: determinar para las empresas el riesgo de sus 
productos en la infección de listeria; incrementar la efectividad de los controles adoptados por las empresas en relación con peligro de listeria; verificar el cumplimiento de los límites de listeria marcados por las autoridades; establecer criterios homogéneos para mejorar la eficacia de los controles oficiales. Por lo que hace al ámbito de aplicación del Plan, éste va dirigido exclusivamente a aquellas empresas del sector cárnico que fabriquen o envasen alimentos listos para el consumo de modo directo, es decir, que no impliquen cocinado o transformación del producto.

Se prevé en el Plan la realización de una visita de inspección en todas las empresas incluidas en su alcance que controlará todos los requisitos mencionados más arriba. Cuando se detecte un caso positivo de listeria por la empresa a través de su sistema de control, la empresa tendrá que adoptar las siguientes medidas: notificación a la autoridad sanitaria; identificación del producto infectado; trazabilidad del producto; listado de clientes y proveedores; comprobación de que la empresa ha adoptado el sistema de análisis de peligros y evaluación de los riesgos.

\section{GONGLUSIÓN}

Siguiendo la estructura anteriormente contemplada para la redacción de este artículo, nos encontramos en condiciones de extraer las siguientes conclusiones:

En primer lugar, la razonabilidad sobre la que se sustentan estas medidas por lo que hace al sector alimentario, tiene lugar en el principio de precaución y en el principio de análisis de riesgos. Estos principios son de naturaleza científica, así que las autoridades administrativas tendrán que actuar o dejarlo de hacer si así lo hacen ver las autoridades técnicas.

La asunción de estos principios en nuestra legislación y jurisprudencia también ha supuesto un cierto traslado decisorio a los operadores alimentarios que, como consecuencia de la más alta tecnificación de la industria alimentaria, existen también mayores riesgos para los consumidores. Estos operadores ya no solo se les recomiendan tomar medidas, sino que tendrán que hacerlo de forma obligada por mandato legal, mediante distintos sistemas. La gestión de riesgos, es decir, la necesidad de estar continuamente controlando y tomando decisiones antes de que se produzca un daño ha sustituido a la antigua máxima de tomar medidas unilaterales y aisladas.

Es importante hacer notar que el principio de precaución se traducirá en una serie de medidas que tomarán las Administraciones Públicas en la fase de gestión del riesgo, que es la última fase del proceso de evaluación de riesgos para eliminar éstos y que es encomendada al poder político. Las medidas a tomar a raíz del principio de precaución serán más leves o moderadas porque se desconoce el riesgo al que nos enfrentamos. 
Otro principio fundamental que viene rubricado en la legislación comunitaria y nacional es el de análisis de riesgos, el cual viene a complementar al principio de precaución. Este principio analiza los riesgos existentes, siendo conscientes las autoridades que tendrán que entrar en escena porque es inviable aspirar a un riesgo "cero". De las tres fases que componen el proceso de evaluación de riesgos, la más importante es la de determinación debido a que será la que incite a que continúe el proceso de evaluación si se considera por la autoridad científica que debe ser así o, de lo contrario, que acabe el proceso en esta fase si se considera que no existe un riesgo de la entidad suficiente como tal para continuar.

La jurisprudencia comunitaria en este punto habilita a los Estados miembros a valorar la decisión que estimen más conveniente, la cual se dirime entre la protección de la salud y la libre circulación de mercancías, pero es muy escrupulosa exigiendo que el proceso de evaluación de riesgos y la aplicación del principio de precaución se realicen correctamente. Por tanto, a pesar de contar los Estados miembros con dicha facultad discrecional, la autoridad sanitaria y científica invitará a las autoridades políticas a adoptar medidas de revelarse evidencias científicas sobre un riesgo y a la Administración Pública no le quedará más remedio que actuar. Sea como fuere, la última palabra en el sistema de gestión del riesgo la tienen los particulares y empresas privadas que si no actúan diligentemente y cumpliendo las normas sanitarias dictadas por las autoridades se les impondrán una sanción administrativa e incluso penal, pero el daño para la población ya estará hecho.

En segundo lugar, no es desdeñable la cantidad de legislación existente, principalmente comunitaria y estatal, que habilita a las autoridades a tomar medidas provisionales. Una particularidad que poseen estas medidas alimentarias es que en la mayoría de las ocasiones no serán medidas provisionales estricto sensu, sino que éstas consistirán en la actuación frente a un riesgo alimentario ya producido o que se va a producir, pero que no recaen sobre una resolución. Son numerosas las normas legales y reglamentarias que legitiman la adopción de medidas por la Administración Pública. Destaca por su amplitud las medidas de emergencia contempladas en el art.54 Reglamento 178/2002, las cuales suponen un "segundo abanico" en caso de que la Comisión no vea oportuno adoptar medidas en un determinado Estado, pero que solo durarán hasta que la Comisión quiera.

En España, son muchas las leyes con las que contamos para tomar medidas. Sin embargo, la principal norma alimentaria ad hoc es la LSAN. Esta norma estatal tiene una clara inspiración comunitaria, como casi todas aquellas que se ocupan del sector alimentario, por cuanto las medidas que se adopten tendrán que hacer respetar los principios del Reglamento 178/2002 que son de aplicación directa. Además, se prefiere una actuación preventiva de los operadores alimentarios durante toda la 
cadena de producción a una actuación de los poderes públicos mediante medidas efectivas y vinculantes cuando el riesgo alimentario sea palpable, como las medidas de emergencia del art.20 LSAN. En un segundo plano y a modo de rescate, también contamos con la LGS y LGSP, que contienen medidas más genéricas no previstas solo para cuando se produzca una alerta alimentaria, sino cuando estemos ante cualquier tipo de riesgo para la salud pública. Como es obvio, las medidas que pueden tomar las Administraciones Públicas no están tasadas, sino que pueden adoptar cualesquiera decisiones a la vista de las circunstancias.

En tercer lugar, la responsabilidad de la Administración Pública se ubica en la CE y en la LRJSP, proclamando ambos instrumentos legales un sistema de responsabilidad objetiva. El daño alimentario, según la legislación más general, tendrá que ser antijurídico, efectivo, evaluable económicamente e individualizable. Si se cumplen estas condiciones, los particulares no tendrán el deber jurídico de soportar el daño. No obstante, en el ámbito alimentario, siendo una rama más técnica como pudiera serlo también la sanidad, la cláusula "estado de los conocimientos científicos y técnicos" marcará la frontera entre lo jurídico y lo antijurídico y, por ende, entre lo indemnizable y no indemnizable. Esta cláusula tiene su origen en una Directiva europea que fue transpuesta a nuestro Ordenamiento jurídico con la Ley de Productos Defectuosos, cuyo art.140 nos viene a decir que se exonerará de responsabilidad al productor si el daño trae su origen en los denominados "riesgos del desarrollo", es decir, aquellos efectos colaterales que acarrea la mejora y el progreso de un determinado producto. Dicha cláusula ha traído demasiada cola doctrinal, pero sea como fuere, se encuentra actualmente presente en nuestra legislación. Por los productos defectuosos, si no existe causa clara de exoneración, tendrá que responder ya sea la Administración, ya sea el productor alimentario en cuestión.

Desde el punto de vista de la responsabilidad de algunas alertas alimentarias que tuvieron en su día una especial incidencia en Andalucía y aún la siguen teniendo desde el punto de vista económico derivado del gran impacto que supuso para cada una de estas industrias alimentarias la declaración de la alerta, el caso del aceite de orujo fue sin duda el más polémico en cuanto si se generó o no responsabilidad.

El caso del "aceite de orujo" sí fue una auténtica alerta alimentaria que permitió tomar por la Administración la medida de la inmovilización de las partidas de aceite de orujo contaminadas ante la presencia de HAP en unas partidas de aceite. La responsabilidad administrativa se cuestiona porque el TS anuló la alerta alimentaria decretada habiéndose producido perjuicios para la empresa durante todo el período que duró la inmovilización cautelar. Sin embargo, esta responsabilidad alimentaria fue denegada principalmente porque la diligencia de la Administración en decretar la alerta alimentaria cuando detectó la sustancia contaminante en el aceite descartó 
la antijuricidad del daño. Hubiera sido más acertado por los Tribunales esgrimir la cláusula de "los conocimientos científicos y técnicos" para eximir de responsabilidad porque la presencia de esta sustancia estuvo motivada por la introducción de nuevas tecnologías en el proceso de elaboración, además de poder haber alegado que el principio de precaución que persigue en definitiva la salud pública justifica la exención de responsabilidad.

Respecto al caso "Magrudis", que es el más reciente, también el más cercano para nosotros y que afortunadamente tuvo una incidencia muy baja para la población gracias a que se contaba con una legislación alimentaria suficiente para hacer frente a la enfermedad. En Andalucía, que fue el territorio donde se originó el brote de listeriosis y también fue el más afectado, la aprobación de un Plan de Choque logró restablecer la situación de normalidad en la Comunidad Autónoma. Este Plan fue dirigido básicamente a las empresas de transformación cárnica, ya que el problema radicó con una alta probabilidad en un defectuoso control de los riesgos y la puesta en el mercado del producto por parte de una concreta empresa de este sector de producción.

A mi juicio, todas las alertas alimentarias que hemos padecido en los últimos años se podrían haber evitado porque, aunque es cierto que estamos expuestos a riesgos nuevos y cada vez más peligrosos por el avance de la tecnología, ya no existía la excusa de que no existe un mandato claro a cada uno de los participantes de la cadena alimentaria sobre lo que se puede hacer y lo que no se debe hacer bajo ningún concepto. En cualquier caso, por muy grande que sea el esfuerzo de las Administraciones Públicas en ordenar directrices en cada momento, si el ánimo lucroso empresarial prevalece, quizá el componente ético en informar cuando se detecte un riesgo no podrá ser posible. 


\section{BIBLIOGRAFÍA}

BUSTO LAGO, J. M., "La responsabilidad civil de las Administraciones Públicas", en L. F. REGLERO CAMPOS y J. M. BUSTOS LAGO (coords.), Tratado de Responsabilidad Civil. Aranzadi, 2014 (recuperado de Aranzadi Digital).

CALLEJA REINA, M. A., "Contagio del ébola en España: ejemplo de cómo no comunicar una crisis", en C. MATEOS MARTÍN y F. J. HERRERO GUTIÉRREZ (coords), La pantalla insomne, 2016.

CAMPOS ACUÑA, M, C., Comentarios a la Ley 40/2015 de Régimen Furídico del Sector Público, El Consultor de los Ayuntamientos, 2017.

ESTEVE PARDO, J., Técnica, riesgo y derecho: tratamiento del riesgo tecnológico en el derecho ambiental. Ariel, 1999.

ESTEVE PARDO, J., "Ciencia y Derecho ante los riesgos para la salud: evaluación, decisión y gestión”, en Documentación administrativa, núm. 265-266 (2003).

GARCÍA RUBIO, F. y FUENTES I GASÓ, J., La responsabilidad de las administraciones públicas tras la nueva ley de régimen jurídico del sector público. Cuatro estudios, Atelier, 2017.

IZQUIERDO CARRASCO, M. y REBOLLO PUIG, M., "El principio de precaución y la defensa de los consumidores", en Documentación administrativa, núm. 265-266 (2003).

MANTECA VALDELANDE, V., "El principio de precaución alimentaria", en Distribución y consumo, núm. 96 (2007).

PRIETO MOLINERO, R. J., "La excepción por estado de la ciencia en España: ¿un camino hacia la innovación segura?", en Práctica de Derecho de Daños, núm. 51 (2007).

SENTÍS CASTAÑO, C., "Ingeniería genética: insuficiencias teóricas y la aplicación del principio de precaución", en Politica y sociedad, vol. 39, núm. 3 (2002).

TOLEDO MARTÍN, A., Las medidas provisionales en el Derecho alimentario, tesis doctoral, Universidad de Navarra (2012). 